\title{
Healthcare-associated infections in paediatric and neonatal wards: A point prevalence survey at four South African hospitals
}

\author{
C Olivier, ${ }^{1}$ 6th-year medical student; H Kunneke, ${ }^{2} \mathrm{MB}$ ChB, FC Paed (SA), MMed (Paed); N O'Connell, ${ }^{3} \mathrm{MB}$ ChB, MMed (Paed); \\ E von Delft, ${ }^{4} \mathrm{MB}$ ChB, FCPaed, MMed (Paed); M Wates, ${ }^{5} \mathrm{MB}$ ChB, BSc, MMed (Paed); A Dramowski, ${ }^{6} \mathrm{MB}$ ChB, FC Paed (SA), \\ MMed (Paed), Cert Paed ID, DCH, PhD \\ ${ }^{1}$ Faculty of Medicine and Health Sciences, Stellenbosch University, Cape Town, South Africa \\ ${ }^{2}$ Department of Paediatrics, Worcester Provincial Hospital, South Africa \\ ${ }^{3}$ Department of Paediatrics, Khayelitsha District Hospital, Cape Town, South Africa \\ ${ }^{4}$ Department of Paediatrics, Paarl Hospital, South Africa \\ ${ }^{5}$ Department of Paediatrics, Karl Bremer Hospital, Cape Town, South Africa \\ ${ }^{6}$ Division of Paediatric Infectious Diseases, Department of Paediatrics and Child Health, Faculty of Medicine and Health Sciences, \\ Stellenbosch University, Cape Town, South Africa
}

Corresponding author: C Olivier(courtney_olivier@hotmail.com)

\begin{abstract}
Background. Healthcare-associated infections (HAIs) cause substantial morbidity, mortality and healthcare costs. The prevalence of neonatal/paediatric HAI at South African (SA) district and regional hospitals is unknown.

Objectives. To document HAI rates, antimicrobial use for HAI, infection prevention staffing, hand hygiene (HH) provisions and HH compliance rates in neonatal and paediatric wards in two district and two regional hospitals in the Western Cape Province, SA.

Methods. An HAI point prevalence survey (PPS) was conducted in neonatal and paediatric wards at two district and two regional hospitals in the Western Cape during December 2016, applying National Healthcare Safety Network HAI definitions. HAI events and antimicrobial therapy active at $08 \mathrm{~h} 00$ on the PPS day and during the preceding 7 days (period prevalence) were documented. Provisions for $\mathrm{HH}$ and $\mathrm{HH}$ compliance rates were observed on each ward using the World Health Organization's HH surveillance tool.

Results. Pooled point and period HAI prevalence were 9.9\% (15/151; 95\% confidence interval (CI) 6 - 15.8) and 12.6\% (19/151; 95\% CI 8 18.9), respectively. Hospital-acquired pneumonia $(5 / 15,33.3 \%)$, bloodstream infection $(3 / 15,20.0 \%)$ and urinary tract infection $(3 / 15$, $20.0 \%)$ were predominant HAI types. Risk factors for HAI were a history of recent hospitalisation $(8 / 19,42.1 \%$ v. $17 / 132,12.9 \%$; $p<0.001)$ and underlying comorbidity (17/19, 89.5\% v. 72/132, 54.5\%; $p<0.004)$. HH provisions (handwash basins/alcohol hand rub) were available and functional. HH compliance was higher in neonatal than in paediatric wards $(125 / 243,51.4 \%$ v. 25/250, 10.0\%; $p<0.001)$. Overall $\mathrm{HH}$ compliance rates were higher among mothers $(46 / 107,43.0 \%)$ than nurses $(73 / 265,27.8 \%)$ and doctors $(29 / 106,27.4 \%)$.

Conclusions. Neonatal and paediatric HAIs are common adverse events at district and regional hospitals. This at-risk population should be prioritised for HAI surveillance and prevention through improved infection prevention practices and HH compliance.

S Afr Med J 2018;108(5):418-422. DOI:10.7196/SAMJ.2018.v108i5.12862
\end{abstract}

Healthcare-associated infections (HAIs) are the most frequent complication of hospitalisation, resulting in increased morbidity, in-hospital mortality and healthcare costs. ${ }^{[1]}$ Hospitalised neonates and children are at high risk for HAI owing to immunological immaturity, frequent handling by caregivers and increased in-hospital exposure to infectious diseases (notably viral respiratory and gastrointestinal pathogens). ${ }^{[1]}$ HAI surveillance is an important tool in quantifying the burden of disease, planning infection prevention interventions and monitoring the impact of these strategies. However, HAI surveillance is seldom conducted in neonates and children in African hospitals, especially in district and regional hospital settings. ${ }^{[2]}$ Furthermore, hospital design, provisions and human resources to implement best practices in infection prevention are limited in South African (SA) public healthcare facilities. ${ }^{[3]}$

From the scant SA literature documenting HAI in hospitalised neonates and children, bloodstream infections (BSIs), urinary tract infections (UTIs) and hospital-acquired pneumonia (HAP) are the most prevalent HAI types. ${ }^{[3-5]}$ In keeping with international literature, important risk factors for HAI in this population include prematurity, intensive care unit admission, malnutrition and the presence of indwelling devices. ${ }^{[3-7]}$ HIV infection and HIV exposure have recently been identified as novel risk factors for HAI in SA children. ${ }^{[4,8]}$

Limited data on neonatal HAI have been reported from three tertiary neonatal centres in SA, with healthcare-associated (HA) BSI rates of 3.9 (Tygerberg Hospital, Cape Town) ${ }^{[9]}$ and 14/1 000 patient days (Charlotte Maxeke Johannesburg Academic Hospital, Johannesburg), ${ }^{[10]}$ and an overall HAI incidence of 21.6/100 admissions at Grey's Hospital, Pietermaritzburg. ${ }^{[11]}$ The prevalence of HAI among hospitalised children in Cape Town was $24 \%,{ }^{[4]}$ in stark contrast to the $5 \%$ rate determined in a recent Europewide paediatric HAI point prevalence survey (PPS). ${ }^{[6]}$ Previous prospective HAI surveillance in $1989^{[5]}$ and an HAI PPS in 2006, ${ }^{[3]}$ conducted in paediatric wards at two Gauteng hospitals, established HAI prevalence rates of $14.3 \%$ and $16 \%$, respectively, with a predominance of gastrointestinal infections, HA pneumonia and HA BSI. Paediatric HAI surveillance in other low- to middle-income countries (LMICs) reported considerable incidence and prevalence density rates of 13.8 per 100 patients at risk in Cambodia, ${ }^{[12]} 29.1$ per 
1000 patient days in Indonesia ${ }^{[13]}$ and 14.7 per 1000 patient days and $21 \%$ in Uganda. ${ }^{[14]}$

There are limited published data on the antimicrobial management of neonatal and paediatric HAI in SA. Unpublished data from two antibiotic consumption PPSs at Tygerberg Hospital, Cape Town, documented that antibiotic use for treatment of HAI constituted 25\% (2012) and 18\% (2015) of all paediatric antibiotic consumption, with carbapenems being the most frequently prescribed agents for HAI (Dr Heather Finlayson, personal communication, 1 June 2017). In addition, few studies have documented the availability of infection prevention staff, the suitability of hospital design (e.g. isolation facilities) and the availability of provisions for infection prevention (e.g. hand hygiene consumables) in neonatal and paediatric wards in SA healthcare facilities. ${ }^{[15]}$

\section{Objective}

We conducted a PPS to document HAI rates, antimicrobial use for HAI, infection prevention staffing, hand hygiene $(\mathrm{HH})$ provisions and $\mathrm{HH}$ compliance rates in neonatal and paediatric wards at two district and two regional hospitals in the Western Cape Province, SA.

\section{Methods}

\section{Study setting and design}

We conducted the PPS in the neonatal and paediatric wards at two district hospitals (Karl Bremer (A) and Khayelitsha (B)) and two regional hospitals (Worcester (C) and Paarl (D)) ( $N=8$ wards). The bed complement in the paediatric and neonatal wards (excluding kangaroo mother care wards) at each facility was as follows: A: $30+16$; B: $32+14$; C: $18+32$ and D: $20+48$. The antenatal HIV prevalence in the Western Cape is $19 \%$, and there is a well-established prevention of mother-to-child HIV transmission programme $(<2 \%$ HIV transmission in 2015). ${ }^{[16]}$

At each facility, a 1-day HAI PPS was conducted, including all patients admitted to the neonatal and paediatric wards at or before $08 \mathrm{~h} 00$ on the survey day. A standardised paper-based data collection tool was used to collect patient demographics, admission diagnoses, HAI events, current antimicrobial prescriptions and microbiology laboratory results. HAI events that met the 2016 Centers for Disease Control and Prevention (CDC)/National Healthcare Network (NHSN) surveillance definitions were included (both active HAI events and HAI events that had occurred in the preceding 7-day period); additional data were collected on HAI type, pathogen (if known), date of HAI onset and HAI management. On the PPS day, the infection prevention and control (IPC) nurse practitioner at each facility was interviewed regarding practices at their institution (IPC audits/assessments, training and challenges, HAI surveillance methods, and reporting of HAI rates/outbreaks to facility management). An audit of $\mathrm{HH}$ provisions and $\mathrm{HH}$ compliance was conducted by direct observation in each ward using the World Health Organization (WHO) tool. ${ }^{[17]}$ The ratio of alcohol hand rub and handwash basins to bed capacity was calculated for the neonatal and paediatric wards at each site.

\section{Study definitions}

The presence and type of HAI event were assessed using the NHSN 2016 definitions and criteria: ${ }^{[1]}$ 'a localized or systemic infective condition in a patient admitted for at least 48 hours (with no evidence that the infection was incubating or present during the time of admission) or a patient who presents with acute infection following a previous health facility admission within the last 30 days'. Bed occupancy (\%) was calculated as the number of admitted patients divided by the number of registered neonatal or paediatric beds.
Antimicrobial use (\%) was calculated as the number of children in each ward receiving any oral or intravenous antimicrobial divided by the total number of patients in the ward multiplied by 100 . $\mathrm{HH}$ compliance (\%) was calculated as number of $\mathrm{HH}$ actions/number of HH opportunities multiplied by 100; HH compliance was audited on each ward over a 30-minute period. In addition, we documented the availability and functionality of the $\mathrm{HH}$ provisions in each neonatal and paediatric ward. For a handwash basin to be considered functional, running water, hand soap and paper towels had to be available at the basin.

\section{Ethics approval}

Ethics approval and waiver of individual informed consent were granted by the Stellenbosch University Health Research Ethics Committee (ref. no. U16/05/011). The research was approved by the Western Cape Provincial Health Research Committee and the facility manager at each hospital (ref. no. WC_2016RP16_572).

\section{Results}

Of the four hospitals, only one had an IPC nurse practitioner whose time was dedicated to IPC duties; in the other hospitals, these nurses had additional duties including occupational health, quality assurance and sterile services department support (Table 1). All four IPC practitioners reportedly offered frequent in-service training to their facility's staff (at weekly, biweekly or monthly intervals). IPC audits and facility-wide $\mathrm{HH}$ audits were conducted at each site at least annually, but only one hospital performed continuous, hospitalwide HAI surveillance. Availability of functional handwash basins and alcohol hand rub was generally adequate, but varied by facility and ward type (neonatal v. paediatric). Few patient isolation rooms were available at any of the sites, and these were mainly located in paediatric wards, with numbers as follows: hospital A: 1 paediatric $v$. 0 neonatal; hospital B: 1 v. 0; hospital C: 2 v. 2; and hospital D: 3 v. 1.

A total of 151 patients were present in the eight wards on the PPS day. Overall, $51.7 \%$ of the cohort (78/151) were male, $3.3 \%(5 / 151)$ were HIV-infected and receiving antiretroviral therapy, 19.9\% (30/151) were HIV-exposed but uninfected, and 11.9\% (18/151) were malnourished ( 5 were severely malnourished, 2 moderately malnourished and 11 underweight for age). Most patients (93.0\%) had been admitted directly to the hospital, but $7.2 \%(n=11)$ had been transferred in from nearby tertiary hospitals (Groote Schuur, Tygerberg and Red Cross War Memorial) and district hospitals (Robertson and Stellenbosch).

Average neonatal bed occupancy was $87 \%$ (range $72-114 \%$ ), with a mean antimicrobial prescribing rate of $39 \%$ (Table 2). The median gestational age for the patients in the neonatal wards $(n=57)$ was 33 weeks (interquartile range (IQR) 28 - 37) and the age range 0 - 38 days. Average paediatric bed occupancy was 66\% (range 38 - 91), with a mean antimicrobial prescribing rate of $56 \%$ (Table 2). The median age of the patients in the paediatric wards $(n=94)$ was 6 months (IQR 10 days - 2 years). A history of recent hospital admission (discharge in the past 30 days) was documented in 25 (26.6\%) of the paediatric patients.

Overall, 15/151 patients (9.9\%; 95\% confidence interval (CI) 6 15.8) had an active HAI event on PPS day; when the 7-day period HAI prevalence definition was used, a further 4 HAI events were identified $(19 / 151,12.6 \%$; 95\% CI 8 - 18.9). HAI event types included HAP $(5 / 15,33.3 \%)$, BSIs $(3 / 15,20.0 \%)$, UTIs $(3 / 15,20.0 \%)$, and one each of surgical site infection, necrotising enterocolitis, conjunctivitis and hospital-acquired gastroenteritis. The prevalence of HAIs was higher among children than neonates $(11 / 94,11.7 \% \mathrm{v}$. $4 / 57,7 \%$; $p=0.41$ ). Predictors of HAI development in this cohort were 
Table 1. IPC services and hand hygiene provisions in neonatal and paediatric wards in the participating facilities

\begin{tabular}{|c|c|c|c|c|}
\hline & \multicolumn{2}{|c|}{ District hospitals } & \multicolumn{2}{|c|}{ Regional hospitals } \\
\hline & Hospital A & Hospital B & Hospital C & Hospital D \\
\hline IPC nurse practitioner* & Shares non-IPC duties & Dedicated to IPC & Shares non-IPC duties & Shares non-IPC duties \\
\hline IPC training of staff (in-service) & $\begin{array}{l}\text { Orientation programme }+ \\
\text { 2-weekly training }\end{array}$ & Monthly training & Weekly training & Weekly training \\
\hline IPC facility audits & Twice yearly & Annually & Twice yearly & Twice yearly \\
\hline Facility $\mathrm{HH}$ audits & Annually & Annually & Annually & Annually \\
\hline Prospective HAI surveillance & No & No & No & Yes \\
\hline \multicolumn{5}{|l|}{ Ratio of HWBs to cots/beds } \\
\hline Neonatal ward & $1 \mathrm{HWB}$ per 5 cots & $1 \mathrm{HWB}$ per 7 cots & $1 \mathrm{HWB}$ per 6 cots & $1 \mathrm{HWB}$ per 5 cots \\
\hline Paediatric ward & 1 HWB per 3 beds & $1 \mathrm{HWB}$ per 4 beds & 1 HWB per 4 beds & $1 \mathrm{HWB}$ per 1 bed \\
\hline $\begin{array}{l}\text { Proportion of functional HWBs, } \\
n / N(\%)\end{array}$ & $9 / 12(75.0)$ & $10 / 10(100)$ & $12 / 12(100)$ & $12 / 13(92.3)$ \\
\hline \multicolumn{5}{|l|}{ Ratio of AHR to cots/beds } \\
\hline Neonatal ward & 1 AHR per cot & 1 AHR per cot & 1 AHR per 3 cots & 1 AHR per cot \\
\hline Paediatric ward & 1 AHR per 3 beds & 1 AHR per bed & 1 AHR per 3 beds & 1 AHR per 3 beds \\
\hline \multicolumn{5}{|l|}{ Isolation rooms, $n$} \\
\hline Neonatal ward & 0 & 0 & 2 & 1 \\
\hline Paediatric ward & 1 & 2 & 1 & 3 \\
\hline $\begin{array}{l}\text { Isolation beds/total neonatal/ } \\
\text { paediatric beds, } n / N(\%)\end{array}$ & $1 / 46(2.2)$ & $2 / 46(4.3)$ & $3 / 50(6.0)$ & $4 / 68(5.9)$ \\
\hline
\end{tabular}

Table 2. HAI prevalence and management in neonatal and paediatric wards in the participating facilities

\begin{tabular}{|c|c|c|c|c|c|}
\hline & \multicolumn{2}{|c|}{ District hospitals } & \multicolumn{2}{|c|}{ Regional hospitals } & \multirow[b]{2}{*}{ Overall } \\
\hline & Hospital A & Hospital B & Hospital C & Hospital D & \\
\hline $\begin{array}{l}\text { Neonatal ward admissions/registered neonatal beds, } \\
n / N \text { (\% occupancy) }\end{array}$ & $12 / 16(75.0)$ & $16 / 14(114.3)$ & $13 / 18(72.2)$ & $16 / 20(80.0)$ & $57 / 68(83.8)$ \\
\hline Neonates receiving antimicrobials, $n / N(\%)$ & $3 / 12(25.0)$ & 9/16 (56.3) & 7/13 (53.8) & $3 / 16(18.8)$ & 22/57 (38.6) \\
\hline $\begin{array}{l}\text { Paediatric ward admissions/registered paediatric beds, } \\
n / N \text { (\% occupancy) }\end{array}$ & $21 / 30(70.0)$ & $25 / 32(78.1)$ & 29/32 (90.6) & $19 / 48(39.6)$ & $94 / 142(66.2)$ \\
\hline Children receiving any antimicrobial, $n / N(\%)$ & $15 / 21(71.4)$ & $15 / 25(60.0)$ & $14 / 29(48.2)$ & 9/19 (47.4) & $53 / 94(56.4)$ \\
\hline Total HAI events (by point prevalence), $n / N(\%)$ & $1 / 33(3.0)$ & $6 / 41(14.6)$ & $7 / 42(16.7)$ & $1 / 35(2.9)$ & 15/151 (9.9) \\
\hline Neonatal & $0 / 12(0)$ & $1 / 16(6.3)$ & $3 / 13(23.1)$ & $0 / 16(0)$ & $4 / 57(7.0)$ \\
\hline Paediatric & $1 / 21(4.8)$ & $5 / 25(20.0)$ & $4 / 29(13.8)$ & $1 / 19(5.3)$ & 11/94 (11.7) \\
\hline HAI events (by period prevalence) & $1+0$ & $6+0$ & $7+1$ & $1+3$ & $19(15+4)$ \\
\hline New antimicrobial prescription for HAI (\%) & 100 & 100 & 100 & 100 & 100 \\
\hline $\begin{array}{l}\text { Appropriate microbiological specimens submitted for } \\
\text { investigation of HAI (\%) }\end{array}$ & 100 & 100 & 86 & 100 & 97 \\
\hline
\end{tabular}

recent hospitalisation $(8 / 19,42.1 \%$ v. $17 / 132,12.9 \%$; $p<0.001)$ and underlying comorbidity or comorbidities $(17 / 19,89.4 \%$ v. $72 / 132$, $54.5 \%$; $p<0.004)$. Although patients who developed HAI $(n=19)$ had lower median age (33 days (IQR 0 - 108) v. 74 days (IQR 0 - 611)), this difference was not statistically significant.

All patients who experienced an HAI event were newly initiated on an antimicrobial, the most frequently used empirical antimicrobials for HAI being piperacillin-tazobactam plus amikacin $(46.7 \%, n=7)$ and third-generation cephalosporins (cefotaxime or ceftriaxone) $(26.7 \%, n=4)$. Meropenem $(n=1)$, ampicillin $(n=1)$, amoxicillinclavulanic acid $(n=1)$ and chloramphenicol $(n=1)$ were the antimicrobials used in the remaining HAI events. Appropriate laboratory specimens had been submitted before commencement of empirical antimicrobials in most cases. Potential HAI pathogens were identified from 6/15 specimens submitted (overall yield $40.0 \%$ ), for bloodstream infections (group B streptococci $n=2$, Staphylococcus aureus $n=1$ ), urinary tract infections (Escherichia coli and Candida albicans $n=1$ each) and a surgical site infection (Escherichia coli $n=1$ ).

A total of $493 \mathrm{HH}$ opportunities were observed in the eight wards (Fig. 1). HH compliance rates were substantially higher in neonatal wards than paediatric wards $(125 / 243,51.4 \%$ v. $25 / 250$, $10 \% ; p<0.001)$. Overall $\mathrm{HH}$ compliance rates were higher among mothers $(45 / 107,42.1 \%)$ than among nurses $(73 / 263,27.8 \%)$, doctors (29/106, 27.4\%) and other staff, including dieticians, radiographers, and speech and hearing therapists $(3 / 17,17.6 \%)$. As assessed using the WHO ' 5 Moments for Hand Hygiene, ${ }^{[17]}$ the most frequently missed $\mathrm{HH}$ opportunities were 'before an aseptic task' (neonates $33.3 \%$ v. paediatrics $12.5 \%$ ) and 'after touching patient surroundings' 


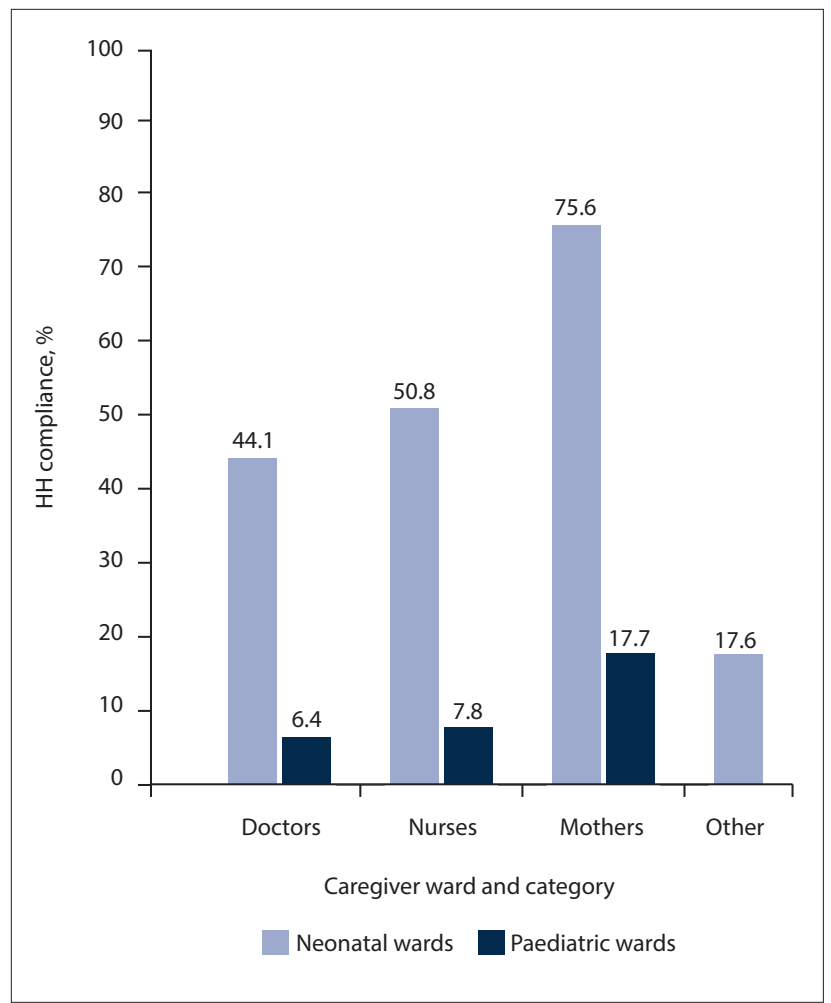

Fig. 1. HH compliance rates (actions/opportunities $\times 100$ ) in neonatal and paediatric wards, by caregiver. Neonatal wards: doctors 26/59 (44.1\%), nurses 62/122 (50.8\%), mothers 34/45 (75.6\%), other staff 3/17 (17.6\%); paediatric wards: doctors $3 / 47$ (6.4\%), nurses 11/141 (7.8\%), mothers 11/62 $(17.7 \%) .(H H=$ hand hygiene; Other = dieticians, radiographers, speech and hearing therapists.)

(neonates $35.9 \%$ v. paediatrics 4.7\%) (Fig. 2). Overall $\mathrm{HH}$ rates per ward type (neonatal v. paediatric) and hospital were as follows: hospital A: $59.3 \%$ v. $17.5 \%$; hospital B: $54 \%$ v. $10.9 \%$; hospital C $39.3 \%$ v. $4.9 \%$; and hospital D: $52.6 \%$ v. $6.5 \%$.

\section{Discussion}

This PPS represents the first estimate of HAI burden in neonatal and paediatric wards in SA regional and district hospitals. We documented an HAI point prevalence rate $(9.9 \%)$ double that reported in a recent Europe-wide neonatal/paediatric HAI PPS, but similar to other LMICs and to that identified by repeated PPS at a tertiary SA children's hospital in 2015/16 (7.4\%). ${ }^{[18]}$

In agreement with SA and international HAI surveillance studies, predominant HAI types were HAP, HA BSI and UTI. ${ }^{[4,6,6]}$ The proportional contribution of HAP to the overall paediatric HAI burden is likely to be under-represented in this cohort, since the PPS was conducted in a summer month. For most HAI events, appropriate laboratory specimens had been submitted prior to empirical antimicrobial prescriptions. Major HA BSI pathogens in SA include K. pneumoniae and S. aureus. ${ }^{[4,9]}$ Although we identified very few bloodstream pathogens in this cohort, $2 / 3$ were group $B$ streptococci bacteraemia events that fulfilled HA BSI criteria but may have represented late-onset maternally derived infection.

Although HIV infection, HIV exposure and malnutrition were frequent in this cohort, these characteristics were not significantly associated with HAI on univariate analysis, possibly owing to the small PPS sample size. Significant risk factors for HAI development (also described in the Tygerberg cohort ${ }^{[4]}$ ) were recent hospitalisation and underlying comorbidity (predominantly prematurity). Development

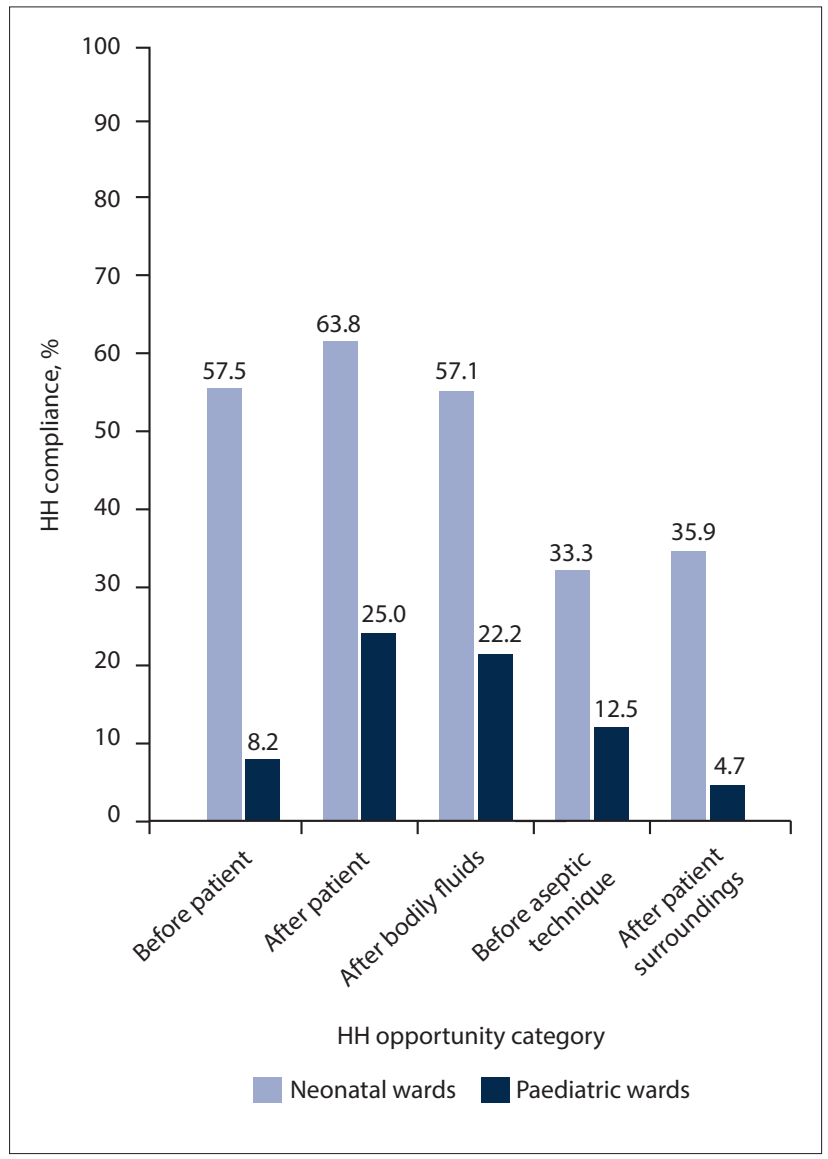

Fig. 2. HH compliance rates (actions/opportunities $\times 100$ ) according to the World Health Organization '5 moments for hand hygiene? ${ }^{3[1]}$ opportunities. Neonatal wards: before patient 42/73 (57.5\%), after patient (44/69 (63.8\%), after patient bodily fluids 8/14 (57.1\%), before aseptic procedure 3/9 (33.3\%), after patient surroundings 28/78 (35.9\%); paediatric wards: before patient $5 / 61$ (8.2\%), after patient 9/36 (25.0\%), after patient bodily fluids 4/18 (22.2\%), before aseptic procedure 1/8 (12.5\%), after patient surroundings 6/127 (4.7\%). (HH = hand hygiene. $)$

of an HAI prompted a new antimicrobial prescription in all $19 \mathrm{HAI}$ events. HAIs are increasingly recognised as major drivers of inpatient antibiotic consumption and therefore potential contributors to antibiotic resistance. Empirical antibiotic selection for HAI management in this cohort included appropriate agents (piperacillintazobactam plus amikacin and third-generation cephalosporins), with no use of carbapenems identified on the PPS day. However, additional longitudinal data on pathogen spectrum and antibiotic susceptibilities are needed from district and regional hospitals to evaluate the appropriateness of empirical HAI treatment regimens, given high prevalence rates of extended-spectrum beta-lactamase (ESBL)-carrying Enterobacteriaceae and methicillin-resistant S.aureus (MRSA). ${ }^{[19]}$ Currently there is no consensus regarding the benchmarking and determination of appropriateness of antibiotic use in neonates and children. Overall neonatal antimicrobial use in our cohort (39\%) was similar to a recent worldwide antimicrobial consumption PPS (36.7\%), ${ }^{[20]}$ but our paediatric antimicrobial use was substantially higher (56\%). However, our paediatric antimicrobial use was lower than that indicated by repeated PPS reporting in Cambodian wards, with a $79 \%$ paediatric antimicrobial use. ${ }^{[11]}$

$\mathrm{HH}$ is a cornerstone of infection prevention in healthcare facilities. In recognising the contribution of $\mathrm{HH}$ to patient safety, the WHO launched the 'Clean care is safer care' campaign in October 2005, 
including guidelines and strategies to increase $\mathrm{HH}$ compliance and reduce HAI rates. ${ }^{[21]}$ The WHO reported that $38 \%$ of LMICs had poor water and sanitation facilities and theorised that higher rates of HAI in LMICs may be due to poor HH resources. ${ }^{[22]}$ In contrast, we reported high availability of alcohol hand rub and functional handwash basins. A simple and cost-effective strategy to improve $\mathrm{HH}$ access and compliance in the neonatal and paediatric wards would be to optimise access to alcohol hand rub even further, providing one bottle of hand rub per bed/cot.

In keeping with other $\mathrm{HH}$ surveillance studies and unpublished annual $\mathrm{HH}$ audits at Tygerberg Hospital (Sister Marina Aucamp, personal communication, 30 June 2017), HH compliance rates were substantially higher in neonatal wards compared with paediatric wards. ${ }^{[23-25]}$ Overall $\mathrm{HH}$ compliance was highest among mothers, with relatively low compliance by doctors and nurses, particularly in the paediatric wards.

Behaviour modification and education on the importance of $\mathrm{HH}$ for healthcare workers and mothers can potentially improve $\mathrm{HH}$ compliance rates. Strategies recommended to improve $\mathrm{HH}$ compliance rates at healthcare facilities include optimal access to $\mathrm{HH}$, patient empowerment regarding healthcare workers' $\mathrm{HH}$, monthly $\mathrm{HH}$ audits with feedback, consequences for non-compliance with $\mathrm{HH}$ recommendations, and continuing education on $\mathrm{HH}$ and HAI (E-learning, symposiums and debates). Potential barriers to $\mathrm{HH}$ compliance, identified by published focus group discussions with registered nurses, include perceived hand damage and high patient workload. ${ }^{[26]}$ Perceived risk of HAI in a particular patient population (e.g. neonates) may be an important factor in healthcare workers' $\mathrm{HH}$ compliance, as suggested by the significantly higher $\mathrm{HH}$ compliance rates noted in all four neonatal wards.

Isolation of patients is an important component in effective IPC, and currently there are limited SA data on paediatric isolation practices. ${ }^{[27]}$ A Tygerberg Hospital-based prospective observational study ${ }^{[27]}$ concluded that $6 \%$ of hospitalised patients were isolated (78\% for IPC purposes), in comparison with 14 - 17\% in high-income countries. The study further highlighted the need for more isolation rooms in low-income settings, particularly for airborne infection transmission prevention (tuberculosis IPC). Although patient isolation rooms were available at each site, no hospital achieved the recommended proportion of isolation beds per ward ( $2-6 \%$ actual $v$. the recommended $20-30 \%) .{ }^{[15]}$

\section{Study limitations}

Limitations of this study included the surveillance method used (PPS), which typically under-estimates HAI burden and is affected by seasonality (HAP events were probably under-represented in this cohort, because the PPS was conducted in a summer month), a small sample size which limited the study's power to identify risk factors for $\mathrm{HAI}$, and direct observation with potential to encourage higher $\mathrm{HH}$ compliance through the Hawthorne effect.

\section{Conclusions}

HAIs are common and preventable inpatient complications that result in significant morbidity, mortality and healthcare costs. Hospitalised neonates and children are a particularly vulnerable population who should be prioritised for HAI surveillance and prevention programmes. Repeated HAI PPSs in district and regional hospitals could be a feasible and useful method to monitor trends in HAI and the impact of HAI prevention strategies. Given the excellent provision for $\mathrm{HH}$ in neonatal/paediatric wards at these hospitals, infection prevention interventions should focus on healthcare worker behaviour modification to improve $\mathrm{HH}$ compliance rates.
Acknowledgements. The authors thank the IPC nurse practitioners at each facility for making themselves available for interviews, as well as the staff and patients at each hospital.

Author contributions. $\mathrm{CO}$ and $\mathrm{AD}$ conceptualised the study, collected and analysed data and produced the first draft of the manuscript. HK, NO'C, EvD and MW provided input on study design and critically revised the manuscript. All authors gave final approval of the version to be published. Funding. Stellenbosch University Undergraduate Research Fund.

Conflicts of interest. None.

1. Horan T, Andrus M, Dudeck M. CDC/NHSN surveillance definition of health-care associated infection and criteria for specific types of infections in the acute care setting. Am J Infect Control 2008;36(5):309and criteria for specific types of infections in the

2. Allegranzi B, Bagheri Nejad S, Combescure C, et al. Burden of endemic health-care-associated infection in developing countries: Systematic review and meta-analysis. Lancet 2011;377(9761):228-241. https:// doi.org/10.1016/S0140-6736(10)61458-4

3. Dusé AG. Infection control in developing countries with particular emphasis on South Africa. South Afr J Epidemiol Infect 2005;20(2):37-41. https://doi.org/10.1080/10158782.2005.11441230

4. Dramowski A, Whitelaw A, Cotton MF. Burden, spectrum and impact of healthcare-associated infections at a South African children's hospital. J Hosp Infect 2016;94(4):364-372. https://doi.org/10.1016/j. jhin.2016.08.022

Cotton M, Berkowitz F, Berkowitz Z, Becker P, Heney C. Nosocomial infections in black South African children. Pediatr Infect Dis J 1989;8(10):676-683. https://doi.org/10.1097/00006454-198910000-00003

6. Zingg W, Hopkins S, Gayet-Ageron A, Holmes A, Sharland M, Suetens C. Health-care-associated infections in neonates, children, and adolescents: An analysis of paediatric data from the European Centre for Disease Prevention and Control point-prevalence survey. Lancet Infect Dis 2017;17(4):381Centre for Disease Prevention and Control point-pre
391. https://doi.org/10.1016/S1473-3099(16)30517-5

7. Magill S, Edwards JR, Bamberg W, et al., Emerging Infections Program Healthcare-associated Infections and Antimicrobial Use Prevalence Survey Team. Multistate point-prevalence survey of healthcare-
and and Antimicrobial Use Prevalence Survey Team. Multistate point-prevalence survey of healthcare-
associated infections. N Engl J Med 2014;370(13):1198-1208. https://doi.org/10.1056/NEJMoal 1306801

8. Archary M, Adler H, la Russa P, Mahabeer P, Bobat R. Bacterial infections in HIV-infected children admitted with severe acute malnutrition in Durban, South Africa. Paediatr Int Child Health 2017;37(1):613. https://doi.org/10.1080/20469047.2016.1198561

9. Dramowski A, Madide A, Bekker A. Neonatal nosocomial bloodstream infections at a referral hospital in a middle-income country: Burden, pathogens, antimicrobial resistance and mortality. Paediatr Int Child Health 2015;35(3):265-272. https://doi.org/10.1179/2046905515Y.0000000029

10. Ballot DE, Nana T, Sriruttan C, Copper PA. Bacterial bloodstream infections in neonates in a developing country. International Scholarly Research Notices. Pediatrics 2012 (2012), Article ID 508512. https://doi. org/10.5402/2012/508512

11. Spicer KB, Green J, Dhada B. Hospital-acquired infections in paediatric medical wards at a tertiary hospital in KwaZulu-Natal, South Africa. Paediatr Int Child Health 2017;38(1):53-59. https://doi.org/1 0.1080/20469047.2017.1299897 12. Stoesser N, Emary K, Soklin S, et al. The value of intermittent point-prevalence surveys of healthcare-
associated infections for evaluation infection control interventions at Angkor Hospital for Children, Siem associated infections for evaluation infection control interventions at Angkor Hospital for Children, Siem
Reap, Cambodia. Trans R Soc Trop Med Hyg 2013;107(4):248-253. https://doi.org/10.1093/trstmh/trt005 Reap, Cambodia. Trans R Soc Trop Med Hyg 2013;107(4):248-253. https://doi.org/10.1093/trstmh/trt005
13. Murni IK, Duke T, Kinney S, et al. Reducing hospital-acquired infections and improving the rational use of antibiotics in a developing country: An effectiveness study. Arch Dis Child 2015;100(5):454. https:/ doi.org/10.1136/archdischild-2014-307297

14. Greco D, Magombe I. Hospital acquired infections in a large north Ugandan hospital. J Prev Med Hyg 2011;52(2):55-58. https://doi.org/10.15167/2421-4248/jpmh2011.52.2.250

5. Dramowski A, Cotton MF, Whitelaw A. Utilization of paediatric isolation facilities in a TB-endemic setting. Antimicrob Resist Infect Control 2015;4:36. https://doi.org/10.1186/s13756-015-0078-z

16. National Department of Health, South Africa. The National Antenatal Sentinel HIV Prevalence Survey South Africa, 2013. http://www.hst.org.za/sites/default/files/DoH\%20Sentinel\%20HIV\%20Survey\%20 High\%20Res\%207102015.pdf (accessed 15 May 2017).

17. World Health Organization. Clean care is safer care: Tools and resources. 2009. http://www.who.int/ gpsc/5may/tools/en/ (accessed 15 May 2017).

18. Dramowski A, Cotton MF, Whitelaw A. Surveillance of healthcare-associated infection in hospitalised South African children: Which method performs best? S Afr Med J 2016;107(1):56-63. https://doi. org/10.7196/SAMJ.2017.v107i1.11431

19. Dramowski A, Cotton MF, Rabie H, et al. Trends in pediatric bloodstream infections at a South African referral hospital. BMC Pediatr 2015;15:33. https://doi.org/10.1186/s12887-015-0354-3

20. Versporten A, Bielicki J, Drapier N, Sharland M, Goossens H, ARPEC project group. The Worldwide Antibiotic Resistance and Prescribing in European Children (ARPEC) point prevalence survey: Developing hospital-quality indicators of antibiotic prescribing for children. J Antimicrob Chemother 2016;71(4):1106-1117. https://doi.org/10.1093/jac/dkv418

21. Pittet D, Allegranzi B, Storr J, et al. Infection control as a major World Health Organization priority for developing countries. J Hosp Infect 2008;68(4):285-292 https://doi.org/10.1016/j.jhin.2007.12.013

22. Horng LM, Unicomb L, Alam A, et al. Healthcare worker and family caregiver hand hygiene in Bangladeshi healthcare facilities: Results from the Bangladesh National Hygiene Baseline Survey. J Hosp Infect 2016;94(3):286-294. https://doi.org/10.1016/j.jhin.2016.08.016

23. Scheithauer $\mathrm{S}$, Oude-Aost $\mathrm{J}$, Heimann $\mathrm{K}$, et al. Hand hygiene in pediatric and neonatal intensive care unit patients: Daily opportunities and infection- and profession-specific analyses of compliance. Am J Infect Control 2011;39(9):732-737. https://doi.org/10.1016/j.ajic.2010.12.020

24. Kirkland KB, Homa KA, Lasky RA, Ptak JA, Taylor EA, Splaine ME. Impact of a hospital-wide hand hygiene initative on healthcare-associated infections: Results of an interrupted time series. BMJ Qual Saf 2012;21(21):1019-1026. https://doi.org/10.1136/bmjqs-2012-000800

5. Wetzker W, Bunte-Schönberger K, Walter J, Pilarski G, Gastmeier P, Reichart CH. Compliance with hand hygiene: Reference data

. White $\mathrm{K}$, Jimmieson N, Obst P, et al. Using a theory of planned behaviour framework to explore han hygiene beliefs at the ' 5 critical moments' among Australian hospital-based nurses. BMC Health Serv Res 2015;15:59. https://doi.org/10.1186/s12913-015-0718-2 